\title{
Impacto académico de las estrategias empleadas en la enseñanza y evaluación de las matemáticas en los primeros semestres de educación superior
}

\author{
Dr. Héctor Manuel Gómez Gutiérrez \\ hmgomez@iteso.mx \\ Departamento de Matemáticas y Física del ITESO \\ División de Ciencias Básicas de Ingeniería del CETI \\ Ingeniería en Desarrollo de Videojuegos de la UAD \\ División de Posgrado en Docencia de UTEG \\ Guadalajara - México
}

\section{RESUMEN}

En el presente trabajo, se desarrolló una investigación documental acerca de las diversas estrategias de enseñanza y aprendizaje de las matemáticas, en donde se ahondó y profundizó en las estrategias de mejores resultados en el aprendizaje, de forma que la construcción del conocimiento cumpla con su objetivo en la asignatura de matemáticas en los primeros semestres de Universidad. En ese sentido, se validó la aplicación de las técnicas, mediante investigación acción participativa, en donde se pusieron grupos piloto, para observar las características o cambios que se plantean, comparando resultados con la enseñanza tradicional. Adicional a lo anterior, se buscaron herramientas especiales para la evaluación de las estrategias empleadas, es decir, evaluar con instrumentos diferentes a un examen, lo que permitió que la orientación de las evaluaciones estuviera sustentada y la estrategia de enseñanza y aprendizaje tuviera un mayor grado de significancia en el sentido de generación o construcción del nuevo conocimiento. Finalmente, se puede concluir en función de los ensayos realizados en los diferentes grupos y Universidades, que el uso de estrategias diferentes, fomenta un incremento sustancial en el aprendizaje por parte de los alumnos y en dependencia directa con la calificación de los mismos, de forma que la evaluación se convierte en un carácter objetivo y significativo hacia los ojos de los alumnos, en donde no solo buscan el pasar, sino que fundamenta el gusto por el aprendizaje y la superación inclusive de forma autogestiva.

Palabras clave: estrategia; enseñanza; aprendizaje; evaluación; herramientas. 


\title{
Academic impact of the strategies used in the teaching and assessment of mathematics in the first semesters of higher education
}

\begin{abstract}
In the present work, a documentary investigation was developed about the various teaching and learning strategies of mathematics, where the strategies for the best results in learning were deepened and deepened, so that the construction of knowledge meets its objective in the subject of mathematics in the first semesters of University. In this sense, the application of the techniques was validated through participatory action research, where pilot groups were set up to observe the characteristics or changes that arise, comparing results with traditional teaching. In addition to the above, special tools were sought for evaluating the strategies used, that is, evaluating with instruments other than an exam, which allowed the orientation of the evaluations to be supported and the teaching and learning strategy had a higher degree of significance in the sense of generation or construction of the new knowledge. Finally, it can be concluded based on the tests carried out in the different groups and Universities, that the use of different strategies promotes a substantial increase in learning by students and in direct dependence on their qualification, in a way that that evaluation becomes an objective and meaningful character in the eyes of the students, where they not only seek to pass, but also establish a taste for learning and self-improvement even in a self-managed way.
\end{abstract}

Keywords: strategy; teaching; learning; evaluation; tools

Artículo recibido: 30 noviembre. 2021 Aceptado para publicación: 29 diciembre 2021

Correspondencia: hmgomez@iteso.mx Conflictos de Interés: Ninguna que declarar 


\section{INTRODUCCIÓN}

A medida que ha evolucionado la forma y estructura de la educación, la enseñanza debe haber experimentado cambios sustanciales para adaptarse a los cambios sociales y tecnológicos que se viven actualmente, es decir, adecuarse a manejar el contexto que los estudiantes tienen presentes, ya que es un eje fundamental el poder estar presentes en la vida del mismo, es decir, partícipes de la realización del estudiante como persona en la sociedad a la que pertenece.

La enseñanza de las matemáticas es un proceso con el cuál se ha incluido un conjunto de debates entre diversos especialistas, debido a que se tienen distintas formas de pensamiento, en donde en una parte, dejan ver que, al ser una ciencia exacta, debe ser mostrada y presentada de forma específica y abstracta, sin tomar en cuenta el contexto de los estudiantes. Por otro lado, distintos especialistas proponen el incluir a las matemáticas como una herramienta para la vida y no como una ciencia totalmente abstracta, es decir, como una ciencia aplicada y aplicable en la vida de cada persona.

Con base a lo anterior, es imperativo analizar ambos puntos de vista, debido a que cada punto sustancial en el proceso de enseñanza, debe contener ambos puntos, pues es imperativo que se conozcan los objetivos generales de la ciencia abstracta y pura y a su vez, conocer en donde se pueden emplear dichos conocimientos específicos, de forma que se pueda vislumbrar la utilidad de los mismos, aún cuando el estudiante no vaya a dedicarse a la ciencia pura, ya que en muchas ramas del conocimiento, se aplican las matemáticas de forma sencilla o abstracta, sin necesidad de aplicarlo como ciencia pura. En concordancia con lo anterior, es fácil ver que el proceso de enseñanza puede tener las dos vertientes fundamentales, pero ante esto, la parte de la evaluación es un factor crucial de este análisis, ya que es imperativo el poder responder a la pregunta ¿cómo debo evaluar las matemáticas?, de acuerdo a la forma pura y abstracta o de acuerdo a la forma aplicada. La realidad es que el uso de nuevas estrategias de evaluación en las que combinan un examen escrito y un proyecto de aplicación, permite en forma clara tomar ambos puntos, pero la realidad es que no siempre se puede manifestar de forma clara estas herramientas, por lo que se busca encontrar puntos adecuados para llevar a cabo dicho proceso de forma eficiente y continua.

En el entorno social actual, la sociedad del conocimiento hace hincapié en la necesidad del uso de estrategias adecuadas para el desarrollo de las competencias de los estudiantes. 
En el área específica de las matemáticas, se debe buscar la implementación de estrategias de enseñanza-aprendizaje que relacionen los contenidos con el contex to inmediato actual. Roman (2015) ha mencionado que la dinámica y la interacción social y pedagógica envuelven de forma sustancial al proceso de enseñanza-aprendizaje, provocando que el aprendizaje de los alumnos se vea claramente afectado por su influencia. Esto deja clara la necesidad de vislumbrar los procesos como un medio de interacción, en donde las relaciones que se vayan obteniendo de los mismos, realizan funciones de procesos cognitivos e influyen a nuestros estudiantes.

Por su parte, Zafra y Martínez (2014) hacen referencia a la relación directa de la sociedad del conocimiento con las tecnologías de la información y la comunicación aplicada en la enseñanza del Cálculo, en donde se ha llegado al cuestionamiento fundamental: “¿qué hace exitosa a un estudiante de Cálculo?” (p. 380). Este cuestionamiento deja necesario el análisis de los procesos tecnológicos que hacen que las competencias de los estudiantes de Cálculo se intensifiquen, es decir, les deja ver cercano algo que parecía inimaginable. El tema de estrategias para la enseñanza del Cálculo Diferencial acordes a la sociedad del conocimiento, tiene por objetivo, el buscar diferentes características en diversas modalidades de estrategias de enseñanza-aprendizaje que favorezcan el desarrollo de competencias en el área de matemáticas. Moreno \& Montoya (2015) proponen estrategias de apoyo para la enseñanza del Cálculo basadas en los entornos virtuales de aprendizaje, mismos que deben cumplir con diversas características para convertirse en un apoyo a la construcción del conocimiento. Los entornos virtuales sirven como una estrategia de enseñanza y aprendizaje, debido a que cumplen con tres requisitos fundamentales, según Moreno \& Montoya (2015):

\section{1) Indagación.}

En este apartado, el estudiante conoce el contenido mediante presentaciones, documentos, animaciones, que le permiten tener acceso al nuevo conocimiento de diferentes maneras, de una forma cercana a él, marcada en su contexto.

\section{2) Interacción.}

En este apartado, el estudiante está en constante comunicación con el docente, con sus compañeros y puede ser de forma síncrona o de forma asíncrona, ya sea por los foros, los chats, las videoconferencias en vivo o grabadas. 


\section{3) Producción}

En este apartado, el estudiante debe poner en acción lo que ha aprendido y desarrollar un producto determinado para demostrar la adquisición de las competencias requeridas en el entorno virtual de aprendizaje. Generalmente es subir un documento, presentación, organizador gráfico a un lugar donde será evaluable en base a una rúbrica.

Por otro lado, Pabón-Gómez (2014) hace mención como estrategia, la implementación de características lúdicas en el desarrollo del aprendizaje haciendo divertido el aprendizaje y por consecuencia, provocando que sea significativo. Esto quiere decir, que los estudiantes sientan que están realizando un juego, sientan que se encuentran inmiscuidos en un ambiente diferente al salón de clase, en donde las características son diferentes a la cotidianeidad. Algunos aprendizajes lúdicos pueden ser como las sopas de letras y los crucigramas, sin embargo, también existen estrategias en donde la dinámica de los salones se transforma en un ambiente diferente, en el que los estudiantes pasan a ser partícipes de su aprendizaje y a la vez actores del mismo.

Ante las diversas herramientas presentes al alcance del profesorado, la selección de estrategias adecuadas para la construcción del conocimiento en el área de matemáticas será el objetivo primordial de este análisis documental. Téliz (2015) propone estrategias innovadoras, en las que se construyen situaciones de enseñanza que propician el desafío del conocimiento de los estudiantes, fomentando la búsqueda y construcción de nuevo conocimiento, este hecho deja claro la necesidad de abrir el panorama a un paradigma diferente a una enseñanza tradicional, propiciando poder llegar a niveles que posiblemente no se encontraban planeados, buscando respuestas a preguntas e inquietudes relacionadas con la temática y que enriquecen en forma pertinente y fehaciente el conocimiento que se está descubriendo por parte de los estudiantes. Los desafíos pueden catalogarse como retos estratégicos para que con aportaciones de los estudiantes se llegue a un conocimiento planeado, pero es importante hacer notar que dicho conocimiento puede salirse de la planeación y llegar más allá, por lo que como docentes debemos incentivar a que se logre el avance y como mínimo se cumplan los objetivos establecidos, pero no poniendo obstáculos a alcanzar nuevos objetivos.

Peña \& Morales (2016) mencionan como estrategia adecuada para la enseñanza de las matemáticas, el uso de la modelación, lo que hace que los problemas comunes de la vida cotidiana, sean relevantes y pertenezcan el mundo de las ciencias exactas. Esta estrategia 
tiene como punto medular el asignar variables a parámetros de la vida cotidiana, tales como el precio de una mercancía, la longitud de un cable, las dimensiones de una figura geométrica que construirá otra figura, entre otros, en donde con base a esa asignación, se pueden proporcionar ecuaciones que determinen un modelo que describe matemáticamente dicho fenómeno, haciendo que el estudiante se motive a adentrarse al mundo de las matemáticas en donde se planteó dicho modelo. Este hecho, da lugar a algunas propuestas para el manejo adecuado y la búsqueda de diferentes estrategias y herramientas que proporcionen el poder llegar a un aprendizaje significativo acorde a la sociedad del conocimiento.

Dentro de algunas estrategias de enseñanza que favorecen la impartición de clases de las matemáticas, Pabón-Gómez (2014) propone la estrategia lúdica pedagógica, misma que se basa en las TIC (Tecnologías de la Información y la Comunicación), que mencionan situaciones contextualizadas y aplicadas al área de las matemáticas. Este relación mencionada, deja clara la visión y se asemeja a los entornos virtuales de aprendizaje mencionados anteriormente, sin embargo, el objetivo de esta aportación, es el uso de diferentes herramientas tecnológicas, tales como software de graficación, como el Quickgraph, Geogebra, Euclides, entre otros, que pueden ayudar en muchos casos a poder observar de forma pertinente la forma que toma la función que ha sido modelada y en base a ello, se determine de forma sustancial la necesidad de vincularse entre sí, ya que la era tecnológica es parte de ellos y ellos de ella, por lo que de la misma forma que se desarrolla, proporciona herramientas sustantivas para la generación de nuevos aprendizajes y nuevas búsquedas de información con base en sus conclusiones o sus representaciones alcanzadas en ellos.

González (2014) plantea como estrategia innovadora el uso de los e-portafolios, las redes sociales y el aprendizaje basado en proyectos, que a su vez, manifiesta la necesidad de una contextualización para su desarrollo e implementación. Con base a esto, los eportafolios se determinan como herramientas virtuales en donde se van guardando de forma pertinente todos los descubrimientos por parte de los estudiantes, construyendo poco a poco los avances en su proceso de aprendizaje, pero almacenados de forma clara en el sitio web de elección del profesor, pudiendo ser una plataforma virtual (como pudiera ser Moodle o Blackboard por mencionar algunas), nube de almacenamiento masivo (como pudiera ser Drive, OneDrive, DropBox, entre otras) o simplemente el uso 
del correo electrónico como centro de entrega. Cuando González (2014) plantea el uso de redes sociales e interacciones, podría hablarse de redes sociales académicas o también de foros de discusión o inclusive el uso y desarrollo de trabajos colaborativos en la nube por medio de Google Docs.

Del mismo modo Caballero \& Bolivar (2015) recomienda hacer a los estudiantes partícipes principales, es decir, hacerlos protagonistas del proceso de enseñanzaaprendizaje. Esto deja clara la necesidad de una planeación específica de cada uno de los procesos, en donde se desenvuelva el estudiante, una correcta organización de los contenidos, en donde el eje sustantivo no sea la exposición del profesor, sino las intervenciones de los alumnos, ya sea en la solución de problemas en el pizarrón, la participación grupal para proporcionar opciones de cómo abordar un problema determinado, de forma que no se encasille una única forma de solución y que ellos determinen de forma creativa y participativa la manera en que les gustaría atacar el problema, en donde el profesor apoye y oriente, pero no de respuestas directas para fomentar la participación estratégica mencionada anteriormente.

Irazoqui \& Medina (2014) mencionan otra manera adecuada, la cuál sería la gamificación de clases o sesiones de trabajo en la que la parte lúdica vuelve a tener presencia en el buen desarrollo de las competencias. Este hecho tiene mucho que ver con el aprendizaje lúdico mencionado anteriormente, ya que vislumbra la realidad en un entorno totalmente diferente al que estaban acostumbrados a tratar en una clase. Cabe señalar que la gamificación presentada por Irazoqui \& Medina (2014) habla de un movimiento total del ambiente, por lo que entorno guiado con música, cambio de roles específicos o inclusive cambio de roles personales serían herramientas para convertir la clase en una estrategia de búsqueda de nuevas realidades, basándose en los conocimientos previos, la interacción con su grupo y sobre todo, la dinámica con la que se lleva dicha estrategia, con la que ningún participante queda fuera del juego y todos se han convertido en el eje preponderante del desarrollo de la actividad.

La mMatemática, en forma general, es una ciencia que determina de forma cualitativa y cuantitativa los cambios o sucesos. Vergel, et al, (2015) hacen mención de que "La matemática es considerada la base de procesos complejos de conocimiento, donde es necesario el pensamiento crítico, reflexivo y analítico" (p. 18). Lo que hace hincapié en la necesidad de provocar el desarrollo específico de esta ciencia de forma contundente, 
pues el desarrollo acorde a la sociedad del conocimiento, es el llegar al punto estratégico en el que la persona sepa reflexionar de forma adecuada, así como analizar el entorno y problema que se presente. Esto deja clara la necesidad del contexto:, la necesidad de hacer que los estudiantes busquen la creatividad para el desarrollo del pensamiento crítico, para poder abordar temáticas centrales en la realidad, que los apoye a reflexionar sobre toma de decisiones y los ayude a analizar los procesos por los que se lleva acabo el avance de la sociedad del conocimiento en función de las herramientas que la mMatemática les ha proporcionado en su realidad.

Robles, et al (2014) menciona que el cCálculo es la ciencia del cambio, basada en la relación entre dos variables que cambian en función una de la otra. Su relación con el contexto, vincula variables y fomenta el modelado de las relaciones matemáticas que en muchos casos se llaman funciones. Pudiendo de esta manera, entender la realidad en base a las matemáticas, tal como Peña \& Morales (2016) lo propusieron anteriormente, es decir, la modelación matemáticas es una estrategia de vital importancia para la enseñanzaaprendizaje, ya que hace hincapié en la habilidad del pensamiento crítico para poder asignar variables determinadas a cada uno de los parámetros necesarios de estudio y análisis, conllevando de esta forma a un aprendizaje significativo, contextual y sobre todo de relación, en el que el estudiante vincula de forma permanente y asocia para sí mismo las reflexiones que fue desarrollando en dicha modelación.

Para implementar una estrategia adecuada a la sociedad del conocimiento, tanto los directivos como los profesores deben de trabajar en un entorno propicio para que este se desarrolle de forma completa. Volante, et. al (2015) menciona que los directivos y profesores requieren de herramientas que favorezcan sus capacidades de innovar en los procesos de fomentar la construcción de conocimiento. Esto quiere decir que, en muchos casos, se puede planear estrategias que vinculen herramientas sustanciales para un aprendizaje enriquecedor, pero los insumos necesarios para llevarse a cabo, no se tienen. Este hecho deja clara la necesidad de una planeación adecuada, de una organización específica por parte de directivos y sobre todo, de apertura para poder visualizar estrategias de enseñanza-aprendizaje diferentes a las anteriores. Esto quiere decir, modificar los métodos tradicionales, por ejemplo, si vas se va a enseñar las tablas de multiplicar con un cartel y repetición, actualizan el aula y mediante el proyector no se debe de proyectar lo que estaba en el cartel, sino buscar herramientas que favorezcan 
dicha interacción, alguna herramienta que deje claro el evento que se desarrolla con la multiplicación, en lugar de solo la repetición. Este hecho es un evento en el que el alumno asocia el conocimiento y al realizarlo, se vincula de forma permanente en su cerebro, a diferencia de que solo sea repetitivo.

González (2014) hace referencia a la necesidad de los aspectos tecnológicos, ya que la sociedad de la información va de la mano con la sociedad del conocimiento. Tal como se ha comentado anteriormente, el uso de herramientas tecnológicas, vincula de forma contundente la relevancia del aprendizaje, ya que deja clara la forma de ver los panoramas involucrados, tales como funciones que son de difícil imaginación y gracias a los avances tecnológicos, puede utilizarse de forma adecuada para poder llevar dicho avance de forma regular a los estándares más altos de calidad de aprendizaje. Del mismo modo, González (2014) también hace hincapié en la utilización de plataformas virtuales como apoyo al aprendizaje presencial, ayudando y complementando lo que se analizó en clase, complementando de forma clase su conocimiento y adquisición del mismo.

El análisis y búsqueda de estrategias de enseñanza-aprendizaje, tiene una necesidad en el conocimiento de las herramientas más importantes que han dado resultado en el proceso de investigación educativa. La búsqueda, clasificación y presentación de su funcionamiento tiene como eje fundamental el proporcionar ideas adecuadas sobre diferentes estrategias válidas para fomentar un aprendizaje significativo del cCálculo dDiferencial, tal como lo menciona Zafra, Vergel y Martínez (2014). Las estrategias que mejor adecúen el proceso de aprendizaje, son de carácter urgente de investigar, ya que proporcionan una herramienta sustancial para que un proceso de aprendizaje se convierta en un proceso exitoso y el conocimiento generado se tenga vislumbrado a través de un pensamiento complejo, que sea reflexivo y que haga que los estudiantes analicen, tal como se mencionó anteriormente. La estrategia es importante, la aceptación de la misma es crucial, ya que, si una estrategia se piensa que es lo mejor para la enseñanza de un tópico, pero los alumnos no la perciben de esta forma, podría deberse a diversas causas, pero las principales serían:

1) Rechazo del tema por no visualizar relación relevante hacia ellos.

2) Sentir que la estrategia no aporta nada o considerarla obsoleta. 
Ambos puntos son importantes, sin embargo, es importante evitar tirar a la basura herramientas buenas, pero en ocasiones hay que desechar lo que ya no es útil hacia el avance del proceso.

Avanzando en el concepto, se puede buscar entender de forma pertinente a lo que se le conoce como estrategia de aprendizaje. Vivas (2010) hace mención de este concepto y lo vincula con otros, comenzando con el concepto de aprendizaje, en donde es vinculado con diversos cambios de conducta, operaciones repetitivas o como se conoce ahora, aprendizaje significativo, en donde se busca un proceso de apropiación y pertenencia. El segundo concepto que toma en cuenta, es la enseñanza, que anteriormente era totalmente de transmisión y en la actualidad es un proceso promotor y dinamizador del aprendizaje. En base a estos dos conceptos, Vivas (2010) hace hincapié en que una estrategia de aprendizaje conlleva una estrategia de enseñanza.

Al profundizar en este concepto, Corredor, Arbelaez \& Pérez (2008) proponen que "el docente no solo orientará sus experiencias dirigidas al desarrollo de competencias cognitivas, actitudinales y axiológicas sino también a competencias metacognitivas.”(p. 157). Esto quiere decir, que las herramientas con que se desenvuelva, deben buscar un equilibrio, ya que la metacognición implica dos puntos muy importantes:

\section{1) Conocer.}

\section{2) Regular.}

Hablando de diferentes áreas del desarrollo académico, Vivas (2010) menciona;

\section{1) Metalectura}

Conocimientos y habilidades que hacen que se pueda leer bien, sabiendo lo que se debe hacer y porqué se debe hacer.

\section{2) Metacomprensión}

Conocimiento de la habilidad que se tiene para comprender fácilmente, así como las habilidades mentales que se aplican en la acción de comprender.

\section{3) Metaignorancia}

Es la ignorancia de la propia ignorancia, es decir, no saber que no se sabe.

\section{4) Meta-atención}

a) Darse cuenta de lo que ya se sabe y de lo que no.

b) Conciencia de la existencia de la tarea y que es relevante atenderla.

c) Jerarquizar los elementos por grado de importancia. 
d) Distribuir el esfuerzo según la importancia.

\section{5) Metamermoria}

Conocimiento de los procesos que implican memorizar o recordar algo.

\section{6) Metaescritura}

Capacidad de revisar, juzgar y corregir lo escrito.

Es importante el desarrollo de estrategias de aprendizaje, que favorezcan la metacognición, sin embargo, es imperativo seleccionar de forma correcta las estrategias a utilizar, tal como Corredor, Arbelaez \& Pérez (2008) proponen: "saber seleccionar la estrategia, que se adecue a cada situación teniendo en cuenta tanto los factores personales como los relacionados con la tarea a ejecutar". Este hecho vuelve a mencionar en forma intrínseca la necesidad de una correcta planeación, en la que los objetivos que se tengan, sean acordes con los resultados que se esperan de la estrategia de aprendizaje utilizada. Un mecanismo diferente, es mencionado por Bain (2008), en el que hace mención de que al ser el fin del profesor el fomentar el aprendizaje, una estrategia fundamental es conocer a sus estudiantes, es decir, identificar aquello que sus estudiantes saben, reconocer la forma en que aprenden, visualizar las diversas formas de comunicación y inmiscuirse en ellas, de tal manera en que pasa a formar parte esencial en la vida de ellos, por lo que esta estrategia se convierte en una constante búsqueda, misma que permite la interacción social antes mencionada y sobre todo, permite al docente una planeación adecuada, que permita de forma contundente y relevante diseñar estrategias de aprendizaje, estrategias de enseñanza y estrategias de evaluación correlacionadas entre sí, en función de las formas adecuadas con las que sus estudiantes aprenden de una forma más eficaz y eficiente.

Del mismo modo Corredor, Arbelaez \& Pérez (2008) hace hincapié en "la importancia de la labor del docente como mediador de los procesos de aprendizaje", lo que combinado con los aportes de Bain (2008), deja clara la visión de planeación del profesorado, pero deja clara la necesidad de intervención social del mismo con respecto a los alumnos, es decir, es indispensable el planear una estrategia de enseñanza para que se convierta en una estrategia de aprendizaje y a su vez tenga su correlación con una correcta estrategia de evaluación vinculada de forma pertinente con las dos anteriores.

Las estrategias de enseñanza y aprendizaje se encuentran divididas en tres diferentes grupos, tal como Huarca et al (2006) propone: 


\section{1) Estrategias para activar o generar conocimientos previos.}

Son estrategias que buscan reencontrar los conocimientos previos de los estudiantes, pudiendo generarlos o crearlos cuando aún no los tienen. Esta estrategia hace relación eficaz a la concordancia que tienen los profesores, pues les ayuda a desarrollar muy bien las demás estrategias en función de lo que conocen sus alumnos.

\section{2) Estrategias para orientar la atención de los estudiantes.}

Son aquellas herramientas o recursos que utiliza el profesor para captar y mantener la atención de los alumnos al momento de estarse llevando a cabo una sesión de aprendizaje.

\section{3) Estrategias para promover el enlace entre los conocimientos previos y la nueva} información que se va a analizar o aprender.

Estas estrategias, son las creadas o diseñadas para crear y potenciar los enlaces que vincular los conocimientos previos o preexistentes con la información o conocimiento nuevo que se va a aprender, fomentando de esta forma un mayor grado de apropiación de los aprendizajes.

A continuación, se mencionarán diversas técnicas, que posteriormente se clasificarán, tal como Pimienta (2012) las propone:

\section{1) Lluvia de Ideas.}

Es una técnica en la que se inicia con una pregunta detonadora relacionada con un tema o problema en particular, conocida comúnmente como una situación problemática. Se espera la participación de los estudiantes, en donde dan su aportación, pero no se profundiza en justificarlo o fundamentarlo. Es importante que todas las ideas expresadas sean validadas. Generalmente el profesor funge como moderador, el cual va anotando en el pizarrón las ideas principales que se van delimitando del tema. Terminada la lluvia de ideas, los conjuntos de ideas son analizados y valorados, por lo que se organizan de acuerdo a la pregunta central y con base en los resultados obtenidos, se llega a una conclusión determinante de los conceptos o problemas planteados. Es importante en esta técnica, realizar una síntesis o conclusión de lo que se obtuvo del análisis y organización de ideas, pues esto puede perderse de no realizarse una concretización de los hechos o temáticas acordados. 


\section{2) Preguntas guía}

Estas preguntas hacen hincapié en buscar diversos detalles específicos sobre algún tema en particular:

a) Causa - ¿por qué?

b) Objetivo - ¿para qué?

c) Concepto - ¿qué?

d) Personaje - ¿quién?

e) Cantidad - ¿cuáto?

f) Lugar - ¿dónde?

g) Tiempo - ¿cuándo?

h) Proceso - ¿cómo?

Un organizador circular puede ayudar a vislumbrar estas preguntas como un conjunto preponderante sobre un tema o problema específico en particular. El profesor se relaciona de forma pertinente como moderador y como ayuda para orientar a los estudiantes en la guía del aprendizaje, pero el estudiante es quien es el partícipe específico del desarrollo de esta estrategia.

\section{3) Estrategia SQA}

Esta estrategia permite al estudiante y al profesor de darse cuenta de los conocimientos previos que se presentan con respecto a un tema, problema o problemática respectiva, incentiva de forma pertinente la necesidad del aprendizaje y la motivación que se tiene.

\section{En forma general, se plantea una tabla de la siguiente forma:}

\begin{tabular}{|c|c|c|}
\hline ¿Qué sé? & ¿Qué quiero saber? & ¿Qué aprendí? \\
\hline $\begin{array}{l}\text { En este apartado, el } \\
\text { estudiante redacta lo que } \\
\text { conoce sobre el tema, } \\
\text { argumentando } \\
\text { conocimientos } \\
\text { respecto al mismo. }\end{array}$ & $\begin{array}{l}\text { En este apartado, el } \\
\text { estudiante, al ver lo que } \\
\text { conoce, redacta lo que le } \\
\text { gustaría conocer con } \\
\text { respecto a la temática } \\
\text { estudiada, de forma que lo } \\
\text { que conoce se } \\
\text { complemente con lo que } \\
\text { quiere entender y } \\
\text { comprender de tema o } \\
\text { problema. }\end{array}$ & $\begin{array}{l}\text { En este apartado, el } \\
\text { estudiante redacta lo que } \\
\text { aprendió del tema, una vez } \\
\text { que fue explicado o } \\
\text { analizado en la sesión de } \\
\text { clase, de forma que se } \\
\text { complementa con las dos } \\
\text { características que se } \\
\text { llenaron previamente al } \\
\text { análisis de los contenidos. } \\
\text { En este apartado se refleja } \\
\text { el aprendizaje nuevo } \\
\text { adquirido por parte de los } \\
\text { estudiantes y se ve si se } \\
\text { alcanzó lo que quería } \\
\text { conocer del tema. }\end{array}$ \\
\hline
\end{tabular}




\section{4) Estrategia Ra-P-Rp}

Esta estrategia, permite visualizar lo que se conoce con respecto a una pregunta específica, de forma anticipada a su desarrollo y posteriormente, una vez analizado el tema, la diferencia de lo que se tenía a lo que se comprendió.

\section{Se sustenta como:}

\section{Pregunta}

\section{Respuesta anterior Respuesta Posterior}

En este apartado, el alumno responde a En este apartado, el estudiante responde a la la pregunta con los conocimientos que pregunta una vez analizado el tema, dejando tiene. Deja clara la forma en que están claro el aprendizaje que se tuvo después de estructurados sus saberes o haber visto el contenido temático. conocimientos previos.

\section{5) Organizadores cognitivos}

Estos organizadores gráficos, permiten acomodar la información de una forma sencilla, de tal manera que los estudiantes van tomando los conceptos más relevantes en función de lo que se está analizando. Cada una de ellas proporciona procesos metacognitivos específicos, basados en la organización, abstracción, relación y correlación de conceptos principales que hacen este tipo de organizadores una herramienta de excelencia, sencilla y de fácil acceso por parte tanto del profesor como del propio estudiante,

\section{Algunos ejemplos de los organizadores cognitivos más importantes son:}

a) Cuadro Sinóptico

b) Diagrama de flujo

c) Diagrama causa y efecto

d) Mapa Conceptual

e) Mapa Mental

f) Diagramas de Venn

g) Línea de tiempo

h) Organigrama circular

i) Organigrama

\section{6) Estrategia PNI}

Es una estrategia que permite plantear el mayor número posible de ideas sobre un evento, suceso o acontecimiento de algo que se observa. 
Algunos ejemplos son:

a) Debate.

b) Mesa Redonda

c) Foro

d) Simposio.

\section{0) Estrategias activas para el desarrollo de competencias}

Son metodologías que pueden o permiten poner en juego una serie de habilidades, capacidades, conocimientos y actitudes en una situación dada y por supuesto en un contexto determinado.

\section{Algunas de ellas son:}

a) Tópico generativo.

b) Simulacro

c) Proyectos.

d) Estudio de caso

e) Aprendizaje basado en problemas

De acuerdo con lo expuesto, el presente estudio documental se enfocó en las siguientes metas: 1) hacer un análisis de las estrategias de enseñanza-aprendizaje desarrolladas en los últimos años; 2) determinar la importancia, la pertinencia y relevancia del uso de cada una de ellas en función de su aplicabilidad; 3) proponer categorías o grados a las estrategias que de acuerdo a este análisis documental cumplan con la función de enriquecer el aprendizaje significativo de las matemáticas. Esto se hará para tener como referencia diversas estrategias aplicables en la enseñanza de las matemáticas que proporcionen resultados adecuados, acordes a la sociedad del conocimiento, así como sus estrategias correlacionadas en su evaluación.

\section{ESTRATEGIAS METODOLÓGICAS O MATERIALES Y MÉTODOS}

En este método, se plantea integrar grupos de alumnos y profesores de 3 diferentes universidades, en donde se trabajará para desarrollar los cambios actualizados en los procesos de enseñanza de las matemáticas y los procesos de evaluación, esperando analizar los resultados correspondientes con los participantes en el mismo.

Se plantea analizar a estudiantes universitarios pertenecientes a las siguientes universidades:

1) Instituto Tecnológico y de Estudios Superiores de Occidente 
2) Universidad de Artes Digitales

3) Centro de Enseñanza Técnica Industrial

La población seleccionada, serán los estudiantes de los primeros tres semestres de las universidades mencionadas anteriormente.

La muestra seleccionada, serán dos grupos del Instituto Tecnológico y de Estudios Superiores de Occidente, de 27 alumnos cada uno, de la materia Cálculo Diferencial. Del mismo modo, tres grupos de la Universidad de Artes Digitales, de las asignaturas Precálculo, Cálculo Diferencial y Cálculo Integral, con 6 alumnos cada uno. Por último serán cuatro grupos del Centro de Enseñanza Técnica Industrial de 40 alumnos, dos de la materia de Precálculo y dos de la materia de Cálculo Diferencial e Integral.

\section{El procedimiento específico tendrá diferentes etapas:}

\section{a) Planeación}

En esta etapa, los profesores tendrán las estrategias previstas para aplicarlas en diferentes etapas de sus cursos, de una forma contundente como actividades relevantes y de gran peso ponderal en su calificación, de forma que se pueda visualizar el avance o retroceso con respecto al logro de los objetivos de aprendizaje.

El catálogo de actividades previstas en función de los logros, está establecido para sus tres fases, pudiendo de la misma manera, apoyarse de entornos virtuales de aprendizaje que complementen la estrategia y fomenten la indagación, interacción y producción por parte de los alumnos (todo esto, será proporcionado al profesor (a) piloto con el que se aplicará el experimento).

\section{b) Aplicación}

Los profesores seleccionados, aplicarán estrategias similares en temáticas similares, con el objetivo de ver y comparar la evolución con respecto a los resultados en el semestre inmediato anterior. Se aplicarán las técnicas en diversas sesiones de trabajo y se registrarán los resultados de la aplicación, es decir, las percepciones del docente y los resultados de productos que obtuvieron los alumnos.

\section{c) Recolección de resultados}

Se evaluarán los productos con base en herramientas de evaluación acordes a la estrategia, que se proveerán posteriormente y se registrarán los resultados obtenidos. 
Se analizará la similitud, mejoría o decremento en los resultados académicos, comparando un parcial con enseñanza tradicional y un parcial con enseñanza implementando estrategias de enseñanza específicas.

Si bien es un dato cualitativo en cuanto a la percepción, se puede tener un dato cuantitativo con respecto a la comparación en dos aspectos, el aspecto del semestre anterior y el aspecto del periodo o parcial anterior, en donde se tengan los resultados académicos presentes y su respectiva comparación.

La relevancia y pertinencia del estudio, se verá siempre y cuando se manifieste su aplicabilidad y las competencias generadas o acrecentadas por parte de los alumnos sean notoriamente superiores a las generadas u obtenidas con una enseñanza tradicional.

\section{RESULTADOS Y DISCUSIÓN}

El reporte ayuda de forma clara a cerrar una estructura de enseñanza, de forma en que se pueda verificar lo que se está aprendiendo y sobre todo, los alcances a los que se puede llegar, que en este caso, son muchos:

- Identificación de las variables.

- Relación de las variables de búsqueda con las presentes.

- Interacción de las variables y los conceptos estudiados.

- Búsqueda de conceptos adicionales para la correcta solución.

- Colaboración y trabajo en equipo.

- Conclusiones y solución óptima y oportuna del proyecto.

Por el lado del aspecto cuantitativo, la comparación de los resultados obtenidos en esta investigación, proporcionan herramientas numéricas que permiten comparar los resultados que se tenían sin estrategias con los resultados que se tuvieron con estrategias. En este caso, $\mathrm{X}$ es el promedio de los alumnos, obtenido como la sumatoria de los valores o calificaciones parciales, llamadas xi y dividido todo entre el número de valores $\mathrm{n}$.

Este valor deja ver el comportamiento general que se tuvo en la muestra analizada.

Por otro lado, la s es la desviación estándar y se calcula sumando las diferencias entre la media aritmética y los valores individuales, divididos entre el número de valores y expuestos a una raíz cuadrada.

Este valor, establece qué tan alejados se encuentran entre sí los valores de la media, si el valor de s es grande, la variación es mucho, pero si el valor de s es pequeño, la variación es poca, por lo que da mayor certeza a los resultados cuando esto ocurre. 


\begin{tabular}{|l|c|c|c|c|c|c|}
\hline \multicolumn{2}{|c|}{ Media y desviación estándar en actividades de activación } \\
\hline \multicolumn{1}{|c|}{ Institución } & $\mathrm{2019B}$ & \multicolumn{2}{c|}{ 2020A } & \multicolumn{2}{c|}{ Diferencia } \\
\hline \multirow{2}{*}{ ITESO } & $\mathrm{X}$ & $\mathrm{s}$ & $\mathrm{X}$ & $\mathrm{s}$ & $\mathrm{X}$ & $\mathrm{x}$ \\
\hline UAD & 73.4 & 5.13 & 80.4 & 4.33 & 7.00 & -0.80 \\
\hline CETI & 81.2 & 4.78 & 84.1 & 3.95 & 2.90 & -0.83 \\
\hline
\end{tabular}

Continuando con las estrategias de atención:

\begin{tabular}{|l|c|c|c|c|c|c|}
\hline Media y desviación estándar en actividades de atención \\
\hline Institución & \multicolumn{2}{|c|}{ 2019B } & \multicolumn{2}{c|}{ 2020A } & \multicolumn{2}{c|}{ Diferencia } \\
\hline & $\mathrm{X}$ & $\mathrm{s}$ & $\mathrm{X}$ & $\mathrm{s}$ & $\mathrm{X}$ & $\mathrm{x}$ \\
\hline ITESO & 77.9 & 4.73 & 81.7 & 3.93 & 3.80 & -0.80 \\
\hline UAD & 75.4 & 6.78 & 82.1 & 5.95 & 6.70 & -0.83 \\
\hline CETI & 73.8 & 4.02 & 80.2 & 4.60 & 6.40 & 0.58 \\
\hline
\end{tabular}

Finalizando con las actividades de abstracción y aplicación:

\begin{tabular}{|l|c|c|c|c|c|c|}
\hline Media y desviación estándar en actividades de abstracción y aplicación \\
\hline Institución & \multicolumn{2}{|c|}{ 2019B } & \multicolumn{2}{c|}{ 2020A } & \multicolumn{2}{c|}{ Diferencia } \\
\hline \multirow{2}{*}{ ITESO } & $\mathrm{X}$ & $\mathrm{s}$ & $\mathrm{X}$ & $\mathrm{s}$ & $\mathrm{X}$ & $\mathrm{x}$ \\
\hline UAD & 71.5 & 4.67 & 81.1 & 5.37 & 9.60 & 0.70 \\
\hline CETI & 80.7 & 5.83 & 84.8 & 4.59 & 4.10 & -1.24 \\
\hline
\end{tabular}


Con base en las tablas anteriores, se presentarán los gráficos especiales que relacionan dichos valores
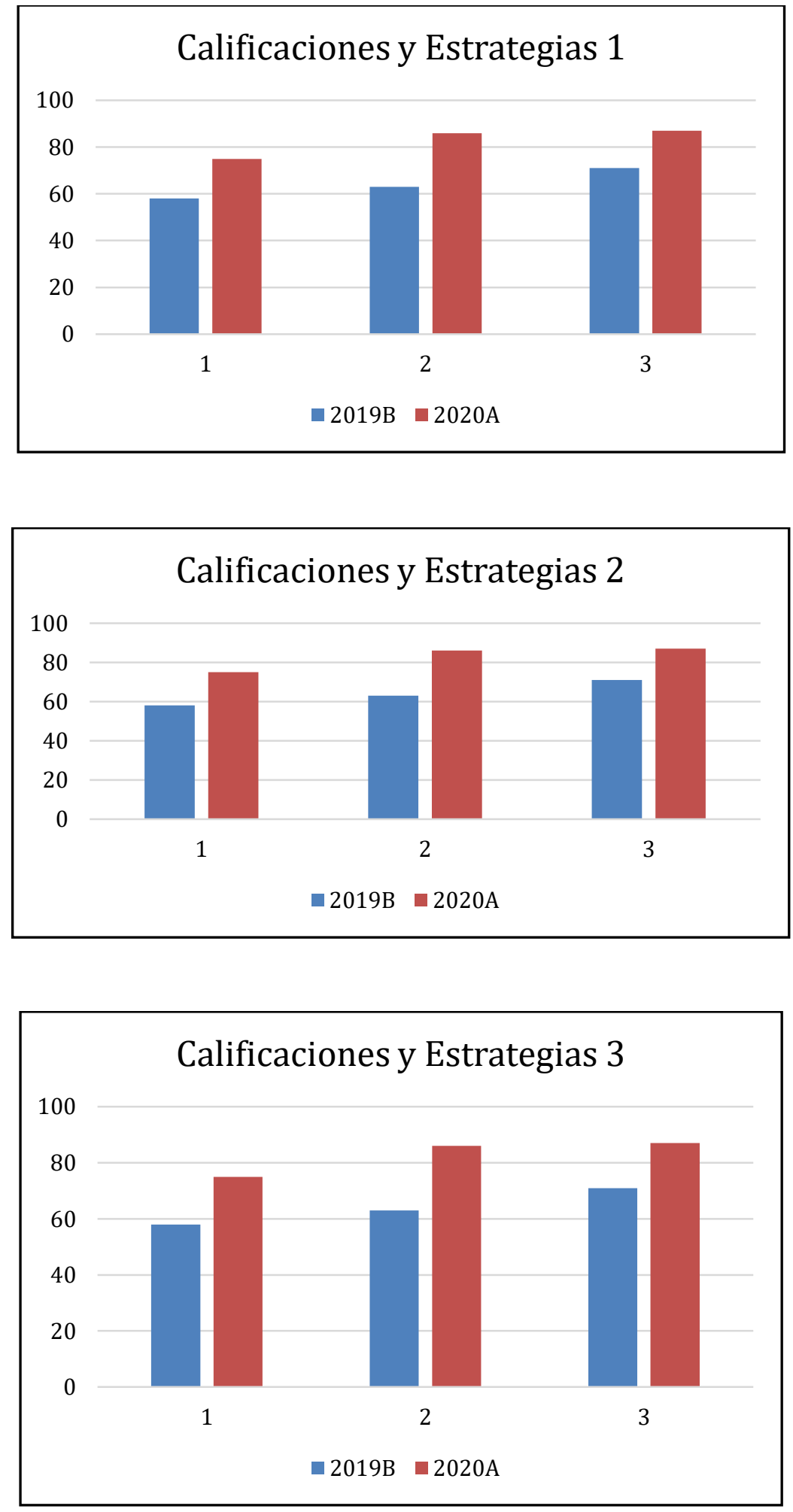

Con base en las tablas y gráficos anteriores, se puede llegar a la conclusión de que las estrategias empleadas para la enseñanza de las matemáticas y su evaluación, varían de 
forma relacional, es decir, cumpliendo requisitos que las vinculan con la acción del experimento, por lo que se puede hacer notar que los resultados de calificación mejoraron a medida que las diferentes estrategias de enseñanza se fueron implementando en el proceso .

Del mismo modo, la evaluación con fundamento en herramientas diversas de evaluación, deja en claro que una evaluación auténtica, integra los tres tipos, diagnóstica, formativa y sumativa, de tal suerte que vincula de forma permanente a las estrategias de enseñanza con sus estrategias de evaluación, es decir, están relacionados de forma directa en su actuar y aplicar.

\section{CONCLUSIÓN O CONSIDERACIONES FINALES}

De acuerdo a lo presentado en el apartado anterior, es fácil ver que las estrategias didácticas tienen un factor preponderante en el aprendizaje significativo de los estudiantes, debido a que el uso e implementación de las mismas, mueven procesos de aprendizaje, vinculan procesos y sobre todo, contrastan los conocimientos previos con los conocimientos nuevos que se van adquiriendo, pudiendo representar este proceso como un ciclo completo del proceso de aprendizaje de los estudiantes, tal como se ve en la figura 6 .

\section{Diagrama de las fases de implementación de estrategias de enseñanza}

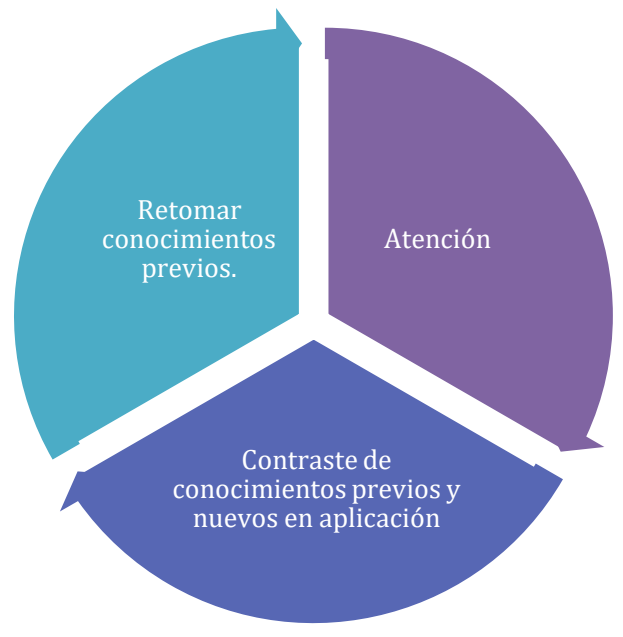

Con base en la investigación, se puede concluir que las estrategias de enseñanza favorecen el aprendizaje de los alumnos, elevando de forma significativa el resultado obtenido por parte de los alumnos, además de que vincula lo aprendido con el contexto y los hace partícipes del mismo. 
Las estrategias de enseñanza más relevantes para la enseñanza de las matemáticas, se implementan de acuerdo a la necesidad de los contenidos y se encuentran englobadas en la figura 7, por lo que sus estrategias e implementación de las mismas, revela resultados y relevancia en los contenidos de forma superior a la clase tradicional que se tenía. En la tabla siguiente, se simplifica la estructura de implementación y acción de dichas estrategias

\begin{tabular}{|l|l|l|}
\hline \multicolumn{1}{|c|}{$\begin{array}{c}\text { Uso y manejo de las estrategias en función de las necesidades de aplicación } \\
\text { Retovios }\end{array}$} & \multicolumn{1}{|c|}{ Atención } & $\begin{array}{c}\text { Contraste entre } \\
\text { conocimientos previos } y \\
\text { nuevos. }\end{array}$ \\
\hline QQQ & Organizadores gráficos & SQA \\
\hline PNI & a) Mapa Conceptual & RaPRp \\
\hline $\begin{array}{l}\text { Organizadores gráficos de } \\
\text { lo que se conoce. }\end{array}$ & b) Mapa Mental & $\begin{array}{l}\text { Proyectos y estrategias } \\
\text { activas }\end{array}$ \\
\hline Debates y mesas redondas & Noticias & ABP \\
\hline
\end{tabular}

El uso y manejo de estas estrategias, ha demostrado una mejoría sustancial en el conocimiento adquirido por parte de los alumnos que fueron parte de la muestra en las tres universidades, revelando que cada una de ellas, aportó de forma sustancial al conocimiento definitivo y proporcionando abstracción especial hacia el desarrollo de actividades importantes, de acuerdo a las cuales, el promedio de los estudiantes fue superior al promedio que obtuvieron estudiantes que trabajaron con exposición magisterial tradicional, sin el uso de estrategias innovadoras del proceso.

Con base a los resultados obtenidos a lo largo de esta investigación, es de importancia preponderante el hacer que los profesores de matemáticas en nivel universitario conozcan los alcances que se tuvo con este desarrollo, ya que el aprendizaje de los estudiantes se incrementa de una forma sustancial, haciendo que los índices de reprobación disminuyan y sobre todo, provocando que el aprendizaje se convierta en un aprendizaje auténtico.

Se recomienda presentar un manual a los profesores de las asignaturas de Cálculo Diferencial en las tres escuelas, en donde se puedan visualizar las estrategias empleadas en algunas temáticas específicas, dando libertad de hacer modificaciones o adecuaciones a las mismas, sin embargo, la implementación de estrategias de enseñanza aprendizaje debe ser por aceptación y no por imposición, de manera que los profesores “crean en la 
estrategia" y con base en ella, propongan herramientas que den a los alumnos las armas necesarias para poder sobrellevar la asignatura y desarrollar su potencial mas allá de lo que se tiene previsto en la misma.

Por otro lado, es importante el combinar las estrategias acordes con los contenidos que se van a llevar a cabo, vinculando de una manera clara la forma en que el alumno ha tenido el acercamiento al concepto determinado, o posiblemente no ha tenido el acercamiento y necesite visualizar documentos, imágenes, gráficos o representaciones específicas que hagan que la estrategia cumpla con su función y de los resultados esperados. Esto quiere decir, que es importante aplicar una una estrategia de atención, cuando se requiere que el estudiante participe de forma activa, en donde necesita todos sus sentidos para poder llevar a cabo una actividad determinada. Por otro lado, cuando se quiere saber el punto de partida que tienen los estudiantes con respecto a un tema específico, se debe tomar en cuenta la estrategia de retomar conocimientos previos, en donde los alumnos plasmarán lo que conocen y con base a ello, se puede avanzar en el contenido. Por último, cuando se tiene un conocimiento previo, se utiliza una estrategia de vinculación de ambos conocimientos, es decir, previos con nuevos, de forma que dichos conocimientos lleven hacia la construcción de un nuevo conocimiento de forma tangible, aplicable y sobre todo contextualizable.

El uso de la evaluación de las estrategias de enseñanza aplicadas en cada uno de sus rubros, debe ser acorde a las necesidades que presenta en el dicho rubro, tal como se presenta en la tabla 10.

Estrategias de evaluación en función de estrategias de aplicación

\begin{tabular}{|c|c|}
\hline Estrategia de enseñanza & Estrategia de evaluación \\
\hline Retomar conocimientos previos & $\begin{array}{l}\text { - Lista de cotejo } \\
\text { - Lista de verificación de } \\
\text { cumplimiento. }\end{array}$ \\
\hline Atención & $\begin{array}{l}\text { - Lista de verificación de } \\
\text { cumplimiento. } \\
\text { - Matriz de evaluación simple }\end{array}$ \\
\hline $\begin{array}{l}\text { Contraste entre conocimientos previos y } \\
\text { conocimientos nuevos }\end{array}$ & $\begin{array}{l}\text { - Matriz de evaluación } \\
\text { - Rúbrica }\end{array}$ \\
\hline
\end{tabular}




\section{LISTA DE REFERENCIAS}

Bain, K. (2008). Lo que hacen los mejores profesores universitarios. Docencia Universitaria. 9, 151-154

Caballero, K. \& Bolivar, A. (2015). El profesorado universitario como docente: hacia una identidad profesional que integre docencia e investigación. Revista de Docencia Universitaria. 13(1). 57-77. doi: 10.4995/redu.2015.6446

Corredor, M. , Arbelaez, L. \& Pérez, I. (2008). Estrategias de enseñanza y aprendizaje. Docencia Universitaria. 9. 155-159

Dryden, G. (2004). La Revolución del aprendizaje. California, E.U.A.: Grupo Editorial Tomo.

González, C. (2014). Estrategias para trabajar la creatividad en la educación superior: pensamiento de diseño, aprendizaje basado en juegos y en proyectos. Revista de Educación a Distancia. 40. 1-15. Recuperado de goo.gl/fWhDXL

Huarca, L., Cortez, R., Bravo, C. \& Verano, W. (2006). Taller de estrategiaspedagógicas. Lima: Editorial San Marcos.

Irazoqui E. \& Medina A. (2014). Aplicación de un diseño curricular modular para la enseñanza del cálculo diferencial. Ingeniare. Revista chilena de ingeniería, 22(4), 576-586. Recuperado de: goo.gl/trpHbq

Moreno, J., \& Montoya, L. (2015). Uso de un entorno virtual de aprendizaje ludificado como estrategia didáctica en un curso de pre-cálculo: Estudio de caso en la Universidad Nacional de Colombia. Revista lbérica de Sistemas e Tecnologias de Informação, 16(12), pp. 1-16. DOI: 10.17013/risti.16.1-16

Ortega-Carbajal, M., Hernández-Mosqueda, J., Tobón-Tobón, S. (2015). Análisis documental de la gestión del conocimiento mediante la cartografía conceptual. Ra Ximhai, 11(4), pp 141-160. Recuperado de https://goo.gl/nWSBxu

Pabón-Gómez, J. (2014). Las TICs y la lúdica como herramientas facilitadoras en el aprendizaje de la matemática. Eco.Mat., 5(1), pp. 37-48. DOI: $10.22463 / 17948231.62$

Peña, L., \& Morales, J. (2016). La modelación matemática como estrategia de enseñanzaaprendizaje: el caso del área bajo la curva. Revista Educación en Ingeniería, 11(21), pp. 64-71.DOI: 10.26507/rei.v11n21.637

Pimienta, J. (2012). Estrategias de enseñanza-aprendizaje. México: Pearson Educación 
Robles. M., Tellechea, E., \& Font, V. (2014). Una propuesta de acercamiento alternativo al teorema fundamental del cálculo. Educación Matemática, 26(2), pp. 69-109. Recuperado de goo.gl/VHtwsV

Rodriguez, R., \& Quiroz, S. (2015). El papel de la tecnología en el proceso de modelación matemática para la enseñanza de las ecuaciones diferenciales. Revista Latinoamericana de Investigación en Matemática Educativa, 19(1), pp. 99-124. DOI: $10.12802 /$ relime.13.1914

Rojas, N., Flores, P., \& Carrillo, J. (2015). Conocimiento Especializado de un Profesor de Matemáticas de Educación Primaria al Enseñar los Números Racionales. Boletim de Educação Matemática, 29(51), pp. 143-166. DOI: 10.1590/1980$4415 \mathrm{v} 29 \mathrm{n} 51 \mathrm{a} 08$

Román, M. (2015). Investigación latinoamericana sobre enseñanza eficaz. Revista Educación y Ciudad. 19. 81-96. Recuperado de goo.gl/FqkyfY

Sheldon, L. (2012). The Multiplayer Classroom. E.U.A.: Cengage Learning

Téliz, F. (2015). Uso didáctico de las TIC en las buenas prácticas de enseñanza de las matemáticas. Estudio de las opiniones y concepciones de docentes de educación secundaria en el departamento de Artigas. Cuadernos de Investigación Educativa, 6(2), pp. 13-31. DOI: 10.18861/cied.2015.6.2.34

Tobón, S. , Guzman, C. Hernández, J. Cardona, S. (2015). Sociedad del conocimiento: Estudio documental desde una perspectiva humanista y compleja. Revista Paradigma. 36(2), pp. 7-36. Recuperado de https://goo.gl/zDZKjg

Vergel, M., Duarte, H., \& Martínez, J. (2015). Desarrollo del pensamiento matemático en estudiantes de cálculo integral su relación con la planificación docente. Revista Cientifica, 23, pp. 17-29. Doi: 10.14483/udistrital.jour.RC.2015.23.a2

Vivas, N. (2010). Estrategias de aprendizaje. Gondola. 5(1), pp. 27-37

Volante, P., Bogolasky, F., Derby, F., \& Gutiérrez, G. (2015). Hacia una teoría de acción en gestión curricular: Estudio de caso de enseñanza secundaria en matemática. Psicoperspectivas Individuo Y Sociedad, 14(2), pp. 96-108. Recuperado de goo.gl/YcQN3T

Werbach, K., \& Hunter, D. (2012). FOR THE WIN How Game Thinking Can Revolutionize your Business. Philadelphia, PA: Wharton Digital Press. 
Impacto académico de las estrategias...

Zafra, L., Vergel, M., \& Martínez, J. (2014). Enseñanza, lenguaje y pensamiento en cálculo. Un análisis cualitativo. Logos, Ciencia \& Tecnología, 5(2), pp. 379-388. DOI: $10.22335 /$ rlct.v5i2.386 